\title{
Interactive comment on "Effects of surface current/wind interaction in an eddy-rich general ocean circulation simulation of the Baltic Sea" by H. Dietze and U. Löptien
}

\section{H. Dietze and U. Löptien}

hdietze@geomar.de

Received and published: 28 June 2016

The referee's comments are typed in bold.

We acknowledge the refee's time and effort!

The two simulations, one with and one without the relative wind correction, result in very different eddy fields. The simulation without the relative wind correction has much higher EKE. As a result of this fundamental difference in the two solution, I do not see a clear path as to how one could use this comparison 
to quantify the influence of the relative wind correction on vertical exchange. This has been one of the primary critaizum of previous efforts to make such comparisons (e.g., Eden and Dietze, 2009). Without some sort of correction to account for the differences between the magnitude of the kinematic variability between the two simulation, the reader is left to wonder if the difference highlighted by the authors are indeed a result of the relative wind correction, or just the manifestation of a (likely) significantly less energetic solution in the simulations including the influence of the surface current on the surface stress. This could be address by redoing the simulations and using some other adjustment to bring the EKE of the two solutions closer together. Another option, and likely the easier one, would be to focus on mesoscale features and how the vertical exchange between them differer in the two simulations. This would also be more in-line with the current and previous research into this topic that highlights the influence of eddy-induced surface currents on imparting a curl in the surface stress.

Only very recently it has become computationally feasible to resolve small-scale surface currents such as coastal currents or part of the mesoscale variability in general ocean circulation model configurations of the Baltic Sea. Among other processes, this - for the first time - introduces a new mechanism: the surface current/wind effect. Theoretical considerations suggest that this should alter the vertical exchange of heat, salt and nutrients substantially. If so, previous models simulations of the Baltic which do not resolve this effect would be flawed. This is of some concern as such simulation are involved in political processes where expensive international decisions concerning future nutrient loads are made. The aim of our manuscript "Effects of surface current/wind interaction in an eddy-rich general ocean circulation of the Baltic Sea" is to explore to what extend earlier model simulations/configurations of the Baltic Sea are potentially biased due to unresolved effects of surface current/wind interaction. We will rephrase part of our introduction such that this becomes clearer. 
The disentangling of (1) the effects of reduced energy input driving less EKE and (2) potentially increased Ekman effects is very interesting - but beyond the scope of our OSD manuscript.

Minor: - The discussion of how these results compare to some of the most important previous works in this field is missing. Once particularly appalling omission is the discussion of how this work builds on the fundamental work by Dewer and Flierl, 1987.

We will include the respective reference in the revised version of the manuscript.

- On page 11, starting at line 4, the authors state that the inclusion of the relative wind correction on the surface stress "does not drive any additional near-surface diapycnal transport ..." This is not surprising as the use of the relative wind generates upwelling and downwelling, which alone, do not drive diapycnal transport. As such, this statement is moot.

We will specify the respective sentence making clear that upwelling and downwelling in combination with typical horizontal diffusive processes and air-sea buoyancy fluxes - do typically drive diapycnal fluxes: e.g. dense water is upwelled to the surface where it is heated by air sea fluxes. Thereby it looses density. When it is subsequently downwelled, the net effect is a diapycnal transport.

Interactive comment on Ocean Sci. Discuss., doi:10.5194/os-2016-12, 2016. 